\title{
Qualitative properties of discrete nonlinear parabolic operators
}

\author{
Róbert Horváth ${ }^{1,4}$. István Faragó ${ }^{2,3,4}$ • János Karátson 1,3,4 \\ Received: 26 February 2019 / Revised: 4 July 2019 / Published online: 30 July 2019 \\ (c) The Author(s) 2019
}

\begin{abstract}
This paper is devoted to the qualitative properties of discretized parabolic operators, such as nonnegativity and nonpositivity preservation, maximum/minimum principles and maximum norm contractivity. In the linear case, earlier papers of the authors (Faragó and Horváth in SIAM Sci Comput 28:2313-2336, 2006, IMA J Numer Anal 29:606-631, 2009) have established the connections between the above qualitative properties and have given sufficient conditions for their validity. The present paper extends the above results to nonlinear discretized parabolic operators, also motivated by the authors' recent paper (Faragó and Horváth in J Math Anal Appl 448:473-497, 2017), which has given related results on the continuous PDE level. A systematic study is presented, ranging from general discrete mesh operators to proper finite element applications.
\end{abstract}

Mathematics Subject Classification 35B50 $\cdot 65 \mathrm{M} 60 \cdot 65 \mathrm{M} 99$

Róbert Horváth

rhorvath@math.bme.hu

István Faragó

faragois@cs.elte.hu

János Karátson

karatson@cs.elte.hu

1 Department of Analysis, Budapest University of Technology and Economics, Egry J. u. 1, Budapest 1111, Hungary

2 Department of Differential Equations, Budapest University of Technology and Economics, Egry J. u. 1, Budapest 1111, Hungary

3 Department of Applied Analysis and Computational Mathematics, Eötvös Loránd University, Pázmány P. stny. 1/c, Budapest 1117, Hungary

4 MTA-ELTE Numerical Analysis and Large Networks Research Group, Pázmány P. stny. 1/c, Budapest 1117, Hungary 


\section{Introduction and preliminaries}

The qualitative properties of the continuous and discrete solutions of partial differential equations are under intensive research nowadays. Namely, beyond the convergence of a numerical method, it is also important to preserve the characteristic qualitative properties of the modeled phenomenon to the numerical solution. This guarantees that the numerical scheme actually used on computers is reliable and efficient. The preservation of the properties can be achieved by a deep analysis of the schemes and it is generally guaranteed with proper assumptions for the spatial discretization and the time-step. For linear parabolic problems the most extensively studied properties are the different maximum and minimum principles, the nonnegativity and nonpositivity preservation and the maximum norm contractivity. Such results can be found in the works $[1,5,7,15,16,20-22,24-27]$ and in the references therein. Not only the preservation of the qualitative properties are important themselves but also their relations. These relations were revealed in an organized framework using discrete mesh operators for linear discrete parabolic problems in an earlier paper of the authors [6].

In the recent decades the interest has turned to the more complicated case of various nonlinear problems in this context, see e.g. $[9,12-14,17,19,23,28]$ where sufficient conditions are given for the qualitative properties, usually related to maximum/minimum principles. However, a study of the relations between such properties has not been carried out yet. Our goal is to give a systematic study on this topic for proper classes of nonlinear problems. Besides the mentioned linear case [5,6], we are motivated by the corresponding background on the continuous PDE level, on which we have derived similar results for certain nonlinear parabolic operators in $[3,8]$.

Let us consider the parabolic operator

$$
\mathcal{N}[u] \equiv \frac{\partial u}{\partial t}-\operatorname{div}(K(x, t, \nabla u))+q(x, t, u)
$$

in the cylinder $\Omega \times(0, T)$, where $\Omega$ is a bounded domain in $\mathbb{R}^{d}$ and $T>0$ is a fixed number. The coefficients $K: \Omega \times(0, T) \times \mathbb{R}^{d} \rightarrow \mathbb{R}^{d}$ and $q: \Omega \times(0, T) \times \mathbb{R} \rightarrow \mathbb{R}$ are given sufficiently smooth functions. We also suppose that $K(x, t, 0)=0$ and $q(x, t, 0)=0$. These conditions are not too restrictive, since the functions $q$ and $K$ generally describe some reaction process and flux quantity, respectively, which vanish in the absence of the given quantity. In [8], we have investigated the qualitative properties of the operator (1) on the continuous level and revealed the implications between its properties. For different versions of minimum and maximum principles (SMP, SBMP, WMP, WBMP), nonnegativity and nonpositivity preservation (NNP, NPP) and maximum norm contractivity (MNC), we have obtained the conditions summarized in Fig. 1. We note that we have simplified the original figure to the case of Dirichlet boundary conditions, and a similar figure applies to minimum principles and nonnegativity preservation.

The goal of this paper is to derive the discrete versions of the above implications and other statements of our previous paper [8]. Thereby we also properly extend our earlier study on the linear parabolic case [5,6], where a diagram analogous to Fig. 1 has been given. We introduce general discrete mesh operators (DMOs), we define their 
Properties of the nonlinear operator $\mathcal{N}[u] \equiv \frac{\partial u}{\partial t}-\nabla(K(x, t, \nabla u))+q(x, t, u)$

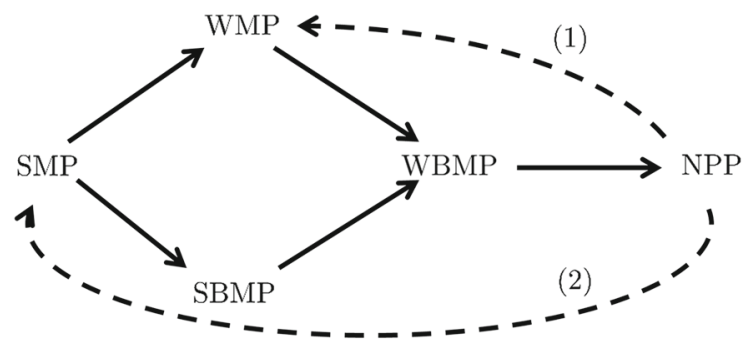

(1) : $\xi \mapsto q(x, t, \xi)$ nondecreasing

(2): $q(x, t, \xi)=0$

Properties of the linearized operator $\mathcal{L}_{z}^{\prime} u \equiv \frac{\partial u}{\partial t}-\nabla\left(K_{\eta}^{\prime}(x, t, \nabla z) \nabla u\right)+q_{\xi}^{\prime}(x, t, z) u$

MNC

NPP, NNP, closed under averaging for both properties NNP and NPP

Fig. 1 Connections between the qualitative properties of the nonlinear parabolic operator (1)

qualitative properties, we characterize the relations between them and give sufficient conditions for the validity of these properties. Then we adapt this study to two-level mesh operators, and finally we apply the results to establish the preservation of the qualitative properties for proper finite element applications.

\section{Nonlinear discrete parabolic mesh operators and the relations between their qualitative properties}

Let $\Omega$ be again a bounded domain in $\mathbb{R}^{d}(d=1,2, \ldots)$ with the usual notation $\partial \Omega$ for its boundary. The space mesh is described by the sets

$$
P=\left\{x_{1}, x_{2}, \ldots, x_{N}\right\} \quad \text { and } \quad P_{\partial}=\left\{x_{N+1}, x_{N+2}, \ldots, x_{N+N_{\partial}}\right\},
$$

consisting of distinct points of $\Omega$ and $\partial \Omega$, respectively. We define $\bar{N}=N+N_{\partial}$ and $\bar{P}=P \cup P_{\partial}$. Let $T$ be again a positive number and $\Delta t$ a positive time-step such that $T=\Delta t n_{T}$ for some positive integer $n_{T}$. For the time mesh we introduce the set $R=\left\{t \in \mathbb{R} \mid t=t_{n}:=n \Delta t, n=0,1, \ldots, n_{T}\right\}$. For any $t \in R$ we introduce the notations

$$
R_{t}:=\{\tau \in R \mid 0<\tau<t\}, \quad R_{\bar{t}}:=\{\tau \in R \mid 0<\tau \leq t\}, \quad R_{\bar{t}}^{0}:=\{\tau \in R \mid 0 \leq \tau \leq t\}
$$


and the sets

$$
Q_{t}=P \times R_{t}, \quad \bar{Q}_{t}=\bar{P} \times R_{\bar{t}}^{0}, \quad Q_{\bar{t}}=P \times R_{\bar{t}}, \quad \Gamma_{t}=\left(P_{\partial} \times R_{\bar{t}}^{0}\right) \cup(P \times\{0\}) .
$$

Definition 1 A mapping from the space of real-valued functions defined on $\bar{Q}_{T}$ to the space of real-valued functions defined on $Q_{T}$ is called a discrete mesh operator (DMO).

Thus, a DMO assigns mesh functions to mesh functions. The domain of a DMO $D$, that is the space of real-valued functions defined on $\bar{Q}_{T}$, is denoted by $\operatorname{dom}(D)$. We define the qualitative properties of DMOs in an analogous way as they were defined for the nonlinear partial differential operator (1) in [8]. Inequalities are understood pointwise on the whole domain of the given mesh function.

Definition 2 A DMO $D$ satisfies

(a) the discrete nonnegativity preservation (DNNP) property if:

$$
v \in \operatorname{dom}(D), \quad t \in R_{T},\left.\quad D[v]\right|_{Q_{\bar{t}}} \geq 0,\left.\quad v\right|_{\Gamma_{t}} \geq 0,\left.\quad \Rightarrow \quad v\right|_{Q_{\bar{t}}} \geq 0
$$

(b) the discrete nonpositivity preservation (DNPP) property if:

$$
v \in \operatorname{dom}(D), \quad t \in R_{T},\left.\quad D[v]\right|_{Q_{\bar{t}}} \leq 0,\left.\quad v\right|_{\Gamma_{t}} \leq 0,\left.\quad \Rightarrow \quad v\right|_{Q_{\bar{t}}} \leq 0
$$

(c) the discrete weak boundary maximum principle (DWBMP) and the discrete strong boundary maximum principle (DSBMP), respectively, if for all $t \in R_{T}$ and $v \in$ $\operatorname{dom}(D)$ with $\left.D[v]\right|_{Q_{\bar{t}}} \leq 0$ :

$$
\left.\max v\right|_{Q_{t}} \leq \begin{cases}\max \left\{0,\left.\max v\right|_{\Gamma_{t}}\right\} & (\text { DWBMP }) \\ \left.\max v\right|_{\Gamma_{t}} & (D S B M P)\end{cases}
$$

(DSBMP means that $v$ attains its maximum on the parabolic boundary, and DWBMP means the same only for a nonnegative maximum);

(d) the discrete weak boundary minimum principle $(D W B m P)$ and the discrete strong boundary minimum principle (DSBmP), respectively, if for all $t \in R_{T}$ and $v \in$ $\operatorname{dom}(D)$ with $\left.D[v]\right|_{Q_{\bar{t}}} \geq 0$ :

$$
\left.\min v\right|_{\bar{Q}_{t}} \geq \begin{cases}\min \left\{0,\left.\min v\right|_{\Gamma_{t}}\right\} & (D W B m P) \\ \left.\min v\right|_{\Gamma_{t}} & (D S B m P)\end{cases}
$$

(DSBmP means that $v$ attains its minimum on the parabolic boundary, and DWBmP means the same only for a nonpositive minimum);

(e) the discrete weak maximum principle $(D W M P)$ and the discrete strong maximum principle (DSMP), respectively, if for all $t \in R_{T}$ and $v \in \operatorname{dom}(D)$ : 


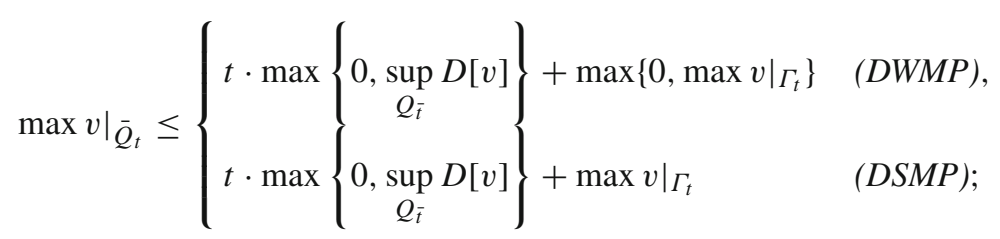

(DWMP and DSMP complete the bound in DWBMP and DSBMP, respectively, with a term including $D[v]$ when the latter has no prescribed sign);

(f) the discrete weak minimum principle $(D W m P)$ and the discrete strong minimum principle $(D S m P)$, respectively, if for all $t \in R_{T}$ and $v \in \operatorname{dom}(D)$ :

$$
\left.\min v\right|_{\bar{Q}_{t}} \geq\left\{\begin{array}{ll}
t \cdot \min \left\{0, \inf _{Q_{\bar{t}}} D[v]\right. \\
t \cdot \min \left\{0, \inf _{Q_{\bar{t}}} D[v]\right.
\end{array}\right\}+\min \left\{0,\left.\min v\right|_{\left.\Gamma_{t}\right\}}(D W m P),\right.
$$

(DWmP and DSmP complete the bound in DWBmP and DSBmP, respectively, with a term including $D[v]$ when the latter has no prescribed sign);

(g) the discrete maximum norm contractivity (DMNC) property if for any $t \in R_{T}$ and any two functions $v_{1}, v_{2} \in \operatorname{dom}(D)$ such that

$$
D\left[v_{1}\right]=D\left[v_{2}\right] \text { in } Q_{\bar{t}},\left.\quad v_{1}\right|_{P_{\partial} \times R_{\bar{t}}^{0}}=\left.v_{2}\right|_{P_{\partial} \times R_{\bar{t}}^{0}},
$$

the relation

$$
\max _{x \in \bar{P}}\left|v_{1}(x, t)-v_{2}(x, t)\right| \leq \max _{x \in \bar{P}}\left|v_{1}(x, 0)-v_{2}(x, 0)\right|
$$

is valid.

Remark 1 (i) The above qualitative properties are formulated for mesh operators similarly to the linear case [6]. The analogous properties for corresponding systems of equations can be formulated in an obvious way. For example, the DNNP simply expresses that nonnegative data yield a nonnegative solution.

(ii) We have defined the maximum and minimum principles separately. It can be checked easily that if a DMO $D$ possesses the property $D[-v]=-D[v]$ (e.g. if $D$ is linear) for all $v \in \operatorname{dom}(D)$ then the maximum principles are equivalent to the corresponding minimum principles and the DNPP is equivalent to the DNNP.

We start with some straightforward relations between the above properties.

Proposition 1 For a DMO, the discrete strong maximum principles DSMP and DSBMP imply the discrete weak maximum principles DWMP, DWBMP, respectively. The discrete maximum principles DSMP and DWMP imply the discrete boundary maximum principles DSBMP and DWBMP, respectively. Similar statements are true for the minimum principles. If the operator satisfies one of the maximum (resp. minimum) principles then it also preserves the nonpositivity (resp. nonnegativity). 
Proof These follow directly from the above definitions.

Now we investigate the implications in the opposite direction, that is we formulate conditions under which the DNPP implies the maximum principles. To this end, we introduce two special grid functions, the constant one $11: \bar{Q}_{T} \rightarrow \mathbb{R}$ and the "function $t " t t \bar{Q}_{T} \rightarrow \mathbb{R}$, respectively:

$$
\mathbb{1}(x, t):=1 \quad \text { for all }(x, t) \in \bar{Q}_{T}, \quad \sharp t(x, t):=t \quad \text { for all }(x, t) \in \bar{Q}_{T} .
$$

The restrictions of these functions to $Q_{T}$ will be denoted in the same way.

Theorem 1 Let a DMO D possess the following property: for all functions $v \in$ $\operatorname{dom}(D)$ and for all nonnegative numbers $\alpha$ and $\beta$, the relation

$$
D[v-\alpha t t-\beta \mathbb{1}] \leq D[v]-\alpha \mathbb{1} \quad(\forall \alpha \geq 0, \beta \geq 0)
$$

is satisfied. Then the DNPP implies the DWMP and the DNNP implies the DWmP.

Proof Assume that the DMO $D$ possesses the DNPP. Let $v$ be a fixed function from $\operatorname{dom}(D)$ and let $t \in R_{T}$ be a fixed value. Let

$$
M_{1}:=\max \left\{0, \sup _{Q_{\bar{t}}} D[v]\right\}, \quad M_{2}:=\max \left\{0,\left.\max v\right|_{\Gamma_{t}}\right\} .
$$

For the DWMP to hold, we must prove that $v(x, \tau) \leq M_{1} t+M_{2}\left(\forall(x, \tau) \in \bar{Q}_{t}\right)$. Let us define the new grid function $\tilde{v}=v-M_{1} t t-M_{2} \mathbb{1}$. Then, based on the assumption of the theorem, we have

$$
D[\tilde{v}]=D\left[v-M_{1} t t-M_{2} \mathbb{1}\right] \leq D[v]-M_{1} \mathbb{1},
$$

which relation shows that $D[\tilde{v}] \leq 0$ on $Q_{\bar{t}}$. Moreover

$$
\tilde{v}=v-M_{1} t t-M_{2}\left\|\leq v-M_{2}\right\| \leq 0
$$

on $\Gamma_{t}$. The discrete nonpositivity preservation property (DNPP) implies that $\tilde{v} \leq 0$ in $Q_{\bar{t}}$, thus also in $\bar{Q}_{t}$, i.e. $v \leq M_{1} t t+M_{2} \leq M_{1} t+M_{2}$ in $\bar{Q}_{t}$ as required.

The other implication regarding the minimum principle can be proven similarly. The values $M_{1}$ and $M_{2}$ must be defined with minimums and infimums, and condition (2) should be applied with the function $v:=v-M_{1} t t-M_{2} 11$ and with the parameters $\alpha=-M_{1}$ and $\beta=-M_{2}$.

Theorem 2 Let a DMO D possess the following property: for all functions $v \in$ $\operatorname{dom}(D)$ and for all nonnegative number $\alpha$ and real number $\beta$, the relation

$$
D[v-\alpha t t-\beta \mathbb{1}] \leq D[v]-\alpha \mathbb{1} \quad(\forall \alpha \geq 0, \beta \in \mathbb{R})
$$

is satisfied. Then the DNPP implies the DSMP and the DNNP implies the DSmP. 
Proof The proof for DSMP is similar to the proof of Theorem 1, because the condition of the theorem guarantees the given estimation independently of the sign of $\beta$. To complete the proof we only need to redefine the parameter $M_{2}$ as $M_{2}:=\left.\max v\right|_{\Gamma_{t}}$. The case of DSmP can be obtained similarly.

Remark 2 Note that the conditions of Theorems 1-2 are generalizations of the conditions obtained for linear DMOs in [6]: $D[11] \geq 0($ resp. $D[1]=0)$ and $D[t t] \geq 1$. This follows simply from $D[v-\alpha t t-\beta 11]=D[v]-\alpha D[t t]-\beta D[\mathbb{1 1}] \leq D[v]-\alpha \mathbb{1}$.

Now we consider the implication of the DMNC property. Similarly to the continuous case in [8], we cannot deduce the DMNC of $D$ directly from the DNNP and DNPP properties of $D$. Instead, we must require the same properties for some linearized version of the operator $D$, the so-called divided difference mesh operator, which is generally used to approximate derivatives.

Theorem 3 Let us suppose that the DMO D satisfies the following assumptions:

(i) $D[v-\beta 11] \leq D[v]$ is satisfied for all nonnegative values $\beta$ and for all functions $v \in \operatorname{dom}(D)$

(ii) for all fixed functions $\bar{w}, \tilde{w} \in \operatorname{dom}(D)$, there exists a $D M O L_{\bar{w}}, \tilde{w}$ that possesses both the DNNP and the DNPP properties, moreover, applying this operator to the function $\bar{w}-\tilde{w}$, we obtain

$$
L_{\bar{w}, \tilde{w}}[\bar{w}-\tilde{w}]=D[\bar{w}]-D[\tilde{w}]
$$

Then the DMO D possesses the DMNC property.

Proof Let us suppose that $v_{1}$ and $v_{2}$ are two arbitrary functions from $\operatorname{dom}(D)$ with the properties

$$
D\left[v_{1}\right]=D\left[v_{2}\right] \text { in } Q_{\bar{t}},\left.\quad v_{1}\right|_{P_{\partial} \times R_{\bar{t}}^{0}}=\left.v_{2}\right|_{P_{\partial} \times R_{\bar{t}}^{0}},
$$

where $t \in R_{T}$ is a fixed number. With the notation $\zeta:=\max _{x \in \bar{P}}\left|v_{1}(x, 0)-v_{2}(x, 0)\right|$ ( $\zeta$ is a nonnegative number) we have to prove that

$$
\max _{x \in \bar{P}}\left|v_{1}(x, t)-v_{2}(x, t)\right| \leq \zeta
$$

Let us consider the function $w^{-}=v_{1}-v_{2}-\zeta \mathbb{1}$. This function is nonpositive on $\Gamma_{t}$. According to assumption (ii), there exists a DMO $L_{v_{1}, v_{2}+\zeta \mathbb{1}}$, such that $L_{v_{1}, v_{2}+\zeta \mathbb{1}}\left[v_{1}-\right.$ $\left.v_{2}-\zeta \mathbb{1}\right]=D\left[v_{1}\right]-D\left[v_{2}+\zeta \mathbb{1}\right] \leq D\left[v_{1}\right]-D\left[v_{2}\right]=0$. Here we applied assumption (i) (with the choices $v=v_{2}+\zeta \mathbb{1}$ and $\beta=\zeta$ ) and condition (4). Thus $L_{v_{1}, v_{2}+\zeta \mathbb{1 1}}\left[w^{-}\right] \leq 0$. Because $L_{v_{1}, v_{2}+\zeta 1}$ is nonpositivity preserving, this implies that $w^{-} \leq 0$ on $Q_{\bar{t}}$ (thus also on $\bar{Q}_{t}$ ), that is

$$
\max _{x \in \bar{P}}\left\{v_{1}(x, t)-v_{2}(x, t)\right\} \leq \zeta .
$$

Similarly, let us consider the function $w^{+}=v_{1}-v_{2}+\zeta 11$. This function is nonneg-

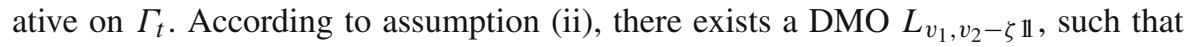
$L_{v_{1}, v_{2}-\zeta \mathbb{1}}\left[v_{1}-v_{2}+\zeta \mathbb{1}\right]=D\left[v_{1}\right]-D\left[v_{2}-\zeta \mathbb{1}\right] \geq D\left[v_{1}\right]-D\left[v_{2}\right]=0$. Here we applied 
Properties of the nonlinear discrete mesh operator $D[v]$

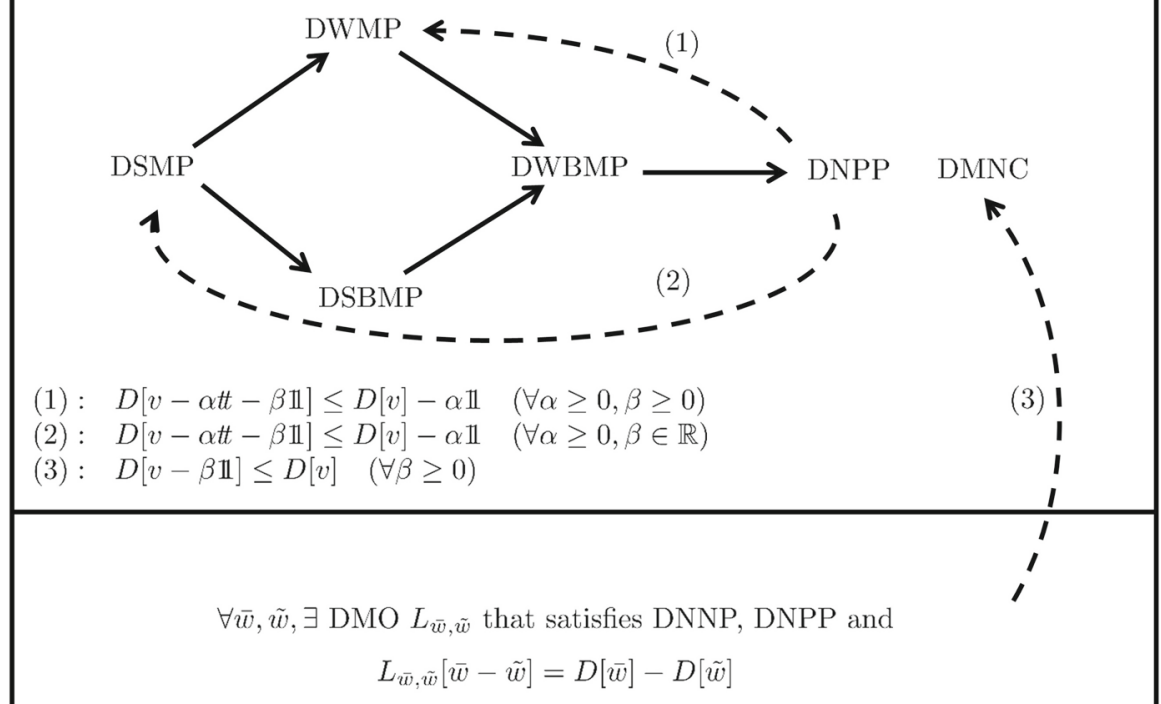

Fig. 2 Connections between the qualitative properties of a discrete nonlinear mesh operator

assumption (i) (with $v=v_{2}$ and $\beta=\zeta$ ) and condition (4). Thus $L_{v_{1}, v_{2}-\zeta \mathbb{1}}\left[w^{+}\right] \geq 0$. Because $L_{v_{1}, v_{2}-\zeta \mathbb{1}}$ is nonnegativity preserving, this implies that $w^{+} \geq 0$ on $Q_{\bar{t}}$ (thus also on $\left.\bar{Q}_{t}\right)$, that is $\max _{x \in \bar{P}}\left\{v_{2}(x, t)-v_{1}(x, t)\right\} \leq \zeta$. This estimate together with (6) shows the required estimate (5).

The implications proven in this section are summarized in Fig. 2. For the discrete minimum principles and the DNNP the figure would be similar.

In the next section, we formulate the above results and conditions for a special type of DMOs: for the so-called two-level DMOs.

\section{Two-level DMOs and the relations between their qualitative properties}

For the sake of simplicity, we denote the value of a mesh function $v$ at the point $\left(x_{i}, t_{n}\right)$ by $v_{i}^{n}$. Moreover, we introduce the column vectors

$$
v^{n}=\left[v_{1}^{n}, \ldots, v_{\bar{N}}^{n}\right]^{T}, \quad v_{0}^{n}=\left[v_{1}^{n}, \ldots, v_{N}^{n}\right]^{T}, \quad v_{\partial}^{n}=\left[v_{N+1}^{n}, \ldots, v_{\bar{N}}^{n}\right]^{T} .
$$

In many numerical solution methods of parabolic partial differential equations, such as finite difference and finite element methods, DMOs appear in the following special form: 


$$
(D[v])_{i}^{n}=\left(X_{1}^{\left(v^{n}\right)} v^{n}-X_{2}^{\left(v^{n-1}\right)} v^{n-1}\right)_{i}, \quad i=1, \ldots, N, \quad n=1, \ldots, n_{T},
$$

where $X_{1}^{\left(v^{n}\right)}, X_{2}^{\left(v^{n-1}\right)} \in \mathbb{R}^{N \times \bar{N}}$ are given matrices. Here the superscripts indicate that the matrices may depend on the vectors $v^{n}$ and $v^{n-1}$, that is on the values of the mesh function $v$ at the time levels $t_{n}$ and $t_{n-1}$, respectively. These matrices may depend also on the index $n$ of the time level and on the time-step $\Delta t$, although for the sake of simplicity we do not indicate this dependence in the notation. Because in the computation of $(D[v])_{i}^{n}$ only the $n$th and $(n-1)$ th time levels are involved, an operator in the form (7) is called a two-level discrete mesh operator (DMO2). To shorten the writing of the formulas, we introduce the formal notation

$$
J\left(w_{1}, w_{2}\right):=X_{1}^{\left(w_{1}\right)} w_{1}-X_{2}^{\left(w_{2}\right)} w_{2} \quad\left(w_{1}, w_{2} \in \mathbb{R}^{\bar{N}}\right) .
$$

Then $(D[v])^{n}=J\left(v^{n}, v^{n-1}\right)$. Our goal is to formulate the conditions of the theorems in the previous section to DMO2s. Let us introduce the column vector $e:=[1, \ldots, 1]^{T} \in \mathbb{R}^{\bar{N}}$. The $N$-element and the $(\bar{N}-N)$-element versions of this vector will be denoted by $e_{0}$ and $e_{\partial}$, respectively.

Theorem 4 Condition (2) can be guaranteed for the DMO2 (7) by imposing the following assumption on the matrices $X_{1}^{(.)}$and $X_{2}^{(.)}$:

(W) $J\left(w_{1}-a e, w_{2}-b e\right) \leq J\left(w_{1}, w_{2}\right)-\frac{a-b}{\Delta t} e_{0}$ for all $w_{1}, w_{2} \in \mathbb{R}^{\bar{N}}$ and for all nonnegative values $a \geq b \geq 0$.

Proof It can be seen easily that condition (2) is equivalent with the condition

$$
J\left(v^{n}-\alpha n \Delta t e-\beta e, v^{n-1}-\alpha(n-1) \Delta t e-\beta e\right) \leq J\left(v^{n}, v^{n-1}\right)-\alpha e_{0}
$$

$\left(\forall v \in \operatorname{dom}(D), n \in\left\{1, \ldots, n_{T}\right\}, \alpha \geq 0, \beta \geq 0\right.$ ) for DMO2s. Thus, let $v$ be an arbitrary fixed mesh function from $\operatorname{dom}(D), n \in\left\{1, \ldots, n_{T}\right\}$ a fixed number, and $\alpha \geq 0, \beta \geq 0$ two fixed nonnegative numbers. Assumption $(W)$ with the choices $a=\alpha n \Delta t+\beta, b=\alpha(n-1) \Delta t+\beta, w_{1}=v^{n}$ and $w_{2}=v^{n-1}$ results in the required condition (8) directly.

Condition (3) can be guaranteed with a stricter condition, where the sign of the parameters $a$ and $b$ is not fixed unlike in condition (W).

Theorem 5 Condition (3) can be guaranteed for the DMO2 (7) by imposing the following assumption on the matrices $X_{1}^{(.)}$and $X_{2}^{(.)}$:

(S) $J\left(w_{1}-a e, w_{2}-b e\right) \leq J\left(w_{1}, w_{2}\right)-\frac{a-b}{\Delta t} e_{0}$ for all $w_{1}, w_{2} \in \mathbb{R}^{\bar{N}}$ and for all values $a \geq b$.

Proof We have to guarantee condition (8) again but now with arbitrary real values $\beta$. The validity of the condition can be proven in a similar way as for the previous theorem. 
The letters $W$ and $S$ in the notations of the above assumptions indicate that these assumptions guarantee the validity of the weak and strong maximum principles, respectively. This is shown by the next theorem.

Theorem 6 If a DMO2 possesses the DNPP property and fulfills condition $(W)$, then it possesses all the weak maximum principles DWMP and DWBMP. If a DMO2 possesses the DNPP property and fulfills condition $(S)$, then it possesses all the maximum principles DWMP, DSMP, DWBMP and DSBMP.

Similarly, if a DMO2 possesses the DNNP property and fulfills condition $(W)$, then it possesses all the weak minimum principles DWmP and DWBmP. If a DMO2 possesses the DNNP property and fulfills condition $(S)$, then it possesses all the minimum principles $D W m P, D S m P, D W B m P$ and $D S B m P$.

Proof It is a direct consequence of the previous theorems.

Remark 3 For linear DMO2s the matrices $X_{1}^{(.)}$and $X_{2}^{(.)}$do not depend on the values of the mesh function $v$. Let us denote these matrices just by $X_{1}$ and $X_{2}$, respectively. In this case condition $(W)$ simplifies to the condition

$$
-a\left(X_{1}-X_{2}\right) e+X_{2}(b-a) e \leq-\frac{a-b}{\Delta t} e_{0}, \quad \forall a \geq b \geq 0
$$

Let us substitute the values of the parameters into the above relation. We obtain

$$
-(\alpha n \Delta t+\beta)\left(X_{1}-X_{2}\right) e+X_{2}(-\alpha \Delta t) e \leq \frac{-\alpha \Delta t}{\Delta t} e_{0}=-\alpha e_{0}, \quad \forall \alpha, \beta \geq 0
$$

(remember that $\alpha$ and $\beta$ are arbitrary nonnegative numbers). This condition is satisfied if $\left(X_{1}-X_{2}\right) e \geq 0$ and $\Delta t\left(n\left(X_{1}-X_{2}\right) e+X_{2} e\right) \geq e_{0}$. Thus we obtained the conditions derived for linear DMO2s in [6].

Condition $(S)$ gives back also the conditions derived in [6] for linear DMO2s: $\left(X_{1}-X_{2}\right) e=0, \Delta t X_{2} e \geq e_{0}$. Namely, condition $(S)$ has the form

$$
-(\alpha n \Delta t+\beta)\left(X_{1}-X_{2}\right) e+X_{2}(-\alpha \Delta t) e \leq-\alpha e_{0}, \quad \forall \alpha \geq 0, \beta \in \mathbb{R},
$$

which is trivially satisfied under the above conditions.

Until this point we have investigated only the implications between certain qualitative properties of DMO2s. Now we give sufficient conditions for the DNNP and DNPP properties. In view of Theorem 6, these conditions together with the condition $(S)$ will guarantee all the maximum-minimum principles.

Let us introduce the following partitions of the matrices $X_{1}^{(.)}$and $X_{2}^{(.)}$:

$$
X_{1}^{(.)}=\left[X_{10}^{(.)} \mid X_{1 \partial}^{(.)}\right], \quad X_{2}^{(.)}=\left[X_{20}^{(.)} \mid X_{2 \partial}^{(.)}\right]
$$

where $X_{10}^{(.)}$and $X_{20}^{(.)}$are square matrices from $\mathbb{R}^{N \times N}$, and $X_{1 \partial}^{(.)}, X_{2 \partial}^{(.)} \in \mathbb{R}^{N \times N_{\partial}}$. 
Theorem 7 Let us suppose that the matrices in the definition of the DMO2 (7) possess the following properties: for any $z \in \mathbb{R}^{\bar{N}}$,

(P1) $z_{\partial} \leq 0, X_{1}^{(z)} z \leq 0 \Rightarrow z_{0} \leq 0$ (resp. $z_{\partial} \geq 0, X_{1}^{(z)} z \geq 0 \Rightarrow z_{0} \geq 0$ ),

(P2) $z \leq 0 \Rightarrow X_{2}^{(z)} z \leq 0$ (resp. $z \geq 0 \Rightarrow X_{2}^{(z)} z \geq 0$ ).

Then DMO2 (7) possesses the DNPP (resp. DNNP) property.

Proof We prove the DNPP case. The DNNP case can be proven similarly. Let $v \in$ $\operatorname{dom}(D)$ and $t \in R_{T}$ with the properties $\left.D[v]\right|_{Q_{\bar{t}}} \leq 0,\left.v\right|_{\Gamma_{t}} \leq 0$. We have to show that $\left.v\right|_{Q_{\bar{f}}} \leq 0$. This implication can be reformulated as follows. We have to show that under the above conditions (P1)-(P2), the conditions $X_{1}^{\left(v^{n}\right)} v^{n}-X_{2}^{\left(v^{n-1}\right)} v^{n-1} \leq 0$, $v^{0} \leq 0, v_{\partial}^{n} \leq 0$ imply $v_{0}^{n} \leq 0(n=1, \ldots, t / \Delta t)$. The nonpositivity of the vectors $v_{0}^{n} \leq 0$ can be shown recursively using the identity

$$
X_{1}^{\left(v^{n}\right)} v^{n}=\left(X_{1}^{\left(v^{n}\right)} v^{n}-X_{2}^{\left(v^{n-1}\right)} v^{n-1}\right)+X_{2}^{\left(v^{n-1}\right)} v^{n-1},
$$

where, due to assumption (P2), the right-hand side is nonpositive. Thus the left-hand side is also nonpositive, and in view of the conditions $v_{\partial}^{n} \leq 0$ and (P1) we obtain that $v_{0}^{n} \leq 0$. This completes the proof.

The following theorem gives joint conditions for DNPP and DNNP that are stronger than $(\mathrm{P} 1)-(\mathrm{P} 2)$ but can be checked more directly.

Theorem 8 If $X_{2}^{(.)} \geq 0, X_{1 \partial}^{(.)} \leq 0$ and $X_{10}^{(.)}$is regular with $\left(X_{10}^{(.)}\right)^{-1} \geq 0$ then the DMO2 (7) possesses the DNPP and DNNP properties.

Proof To apply Theorem 7, we check that assumptions (P1)-(P2) are satisfied. Indeed, the validity of (P2) is trivial. Moreover, condition (P1) is obtained in the following way. The nonpositivity of $X_{1}^{(z)} z=X_{10}^{(z)} z_{0}+X_{1 \partial}^{(z)} z_{\partial}$ and the relation $X_{1 \partial}^{(z)} z_{\partial} \geq 0$ gives the relation $X_{10}^{(z)} z_{0} \leq 0$. The nonpositivity of $z_{0}$ can be seen after multiplication with the nonnegative inverse matrix $\left(X_{10}^{(z)}\right)^{-1}$. Finally, the same arguments apply with reversed signs as well.

We close this section with the reformulation of the condition that guarantees the DMNC property for DMO2s.

Theorem 9 Let us suppose that the DMO2 D satisfies the following assumptions:

$\left(W_{=}\right) \quad J\left(w_{1}-a e, w_{2}-a e\right) \leq J\left(w_{1}, w_{2}\right)$ for all $w_{1}, w_{2} \in \mathbb{R}^{\bar{N}}$ and for all nonnegative values $a \geq 0$;

(L) for all fixed functions $\bar{w}, \tilde{w} \in \operatorname{dom}(D)$, there exists a DMO $L_{\bar{w}, \tilde{w}}$ that possesses both the DNNP and the DNPP properties, moreover applying this operator to the function $\bar{w}-\tilde{w}$ we obtain

$$
L_{\bar{w}, \tilde{w}}[\bar{w}-\tilde{w}]=D[\bar{w}]-D[\tilde{w}]
$$

Then the DMO2 (7) possesses the DMNC property. 
Proof Choosing $a=\beta$ and using the form (7) of a DMO2, we obtain the conditions of Theorem 3.

Remark 4 We used the equality sign in the subscript because this condition can be obtained from condition (W) with the setting $a=b$. Condition (L) is the same as condition (ii) in Theorem 3.

\section{Relations between the qualitative properties of the finite element discretization of a nonlinear parabolic problem}

In this section we apply the results of the previous section to the finite element (FE) solution of a nonlinear parabolic problem. We consider the problem $\mathcal{N}[u]=f$, where $\mathcal{N}$ is the nonlinear operator (1) and $f: Q_{T} \rightarrow \mathbb{R}$ is a given continuous function. We will characterize the relations of the qualitative properties of these finite element solutions, and formulate conditions that guarantee their validity.

\subsection{Formulation and preliminaries}

First we rewrite the equation $\mathcal{N}[u]=f$ in order to have proper product forms. Let us define

$$
\begin{aligned}
\widetilde{r}(x, t, \xi, \bar{\xi}) & :=\int_{0}^{1} \partial_{3} q(x, t, s \xi+(1-s) \bar{\xi}) \mathrm{d} s, \\
\widetilde{A}(x, t, \eta, \bar{\eta}) & :=\left[\int_{0}^{1} \partial_{3_{j}} K_{k}(x, t, s \eta+(1-s) \bar{\eta}) \mathrm{d} s\right]_{k, j=1, \ldots, d},
\end{aligned}
$$

where $\partial_{3}$ denotes the derivative w.r.t. the third argument of the function $q$, further, $K_{k}$ is the $k$ th coordinate function of the vector function $K$ and $\partial_{3_{j}}$ denotes the partial derivative according to the $j$ th coordinate of the third argument of $K$. Using the Newton-Leibniz formula, we have

$$
\widetilde{r}(x, t, \xi, \bar{\xi})(\xi-\bar{\xi})=q(x, t, \xi)-q(x, t, \bar{\xi})
$$

Substituting $\bar{\xi}=0$ into the above expression, we obtain

$$
r(x, t, \xi) \xi=q(x, t, \xi)-q(x, t, 0)=q(x, t, \xi),
$$

where we used the simplified notation $r(x, t, \xi):=\widetilde{r}(x, t, \xi, 0)$ and applied the assumption $q(x, t, 0)=0$. Note that if $q$ is nondecreasing w.r.t. $\xi$ then $r \geq 0$. A similar procedure can be carried out for the vector function $K$, using the $d \times d$ matrix function $\widetilde{A}$ :

$$
\begin{aligned}
\widetilde{A}(x, t, \eta, \bar{\eta})(\eta-\bar{\eta}) & =\left[\int_{0}^{1} \frac{\mathrm{d}}{\mathrm{d} s} K_{k}(x, t, s \eta+(1-s) \bar{\eta}) \mathrm{d} s\right]_{k=1, \ldots, d} \\
& =K(x, t, \eta)-K(x, t, \bar{\eta}) .
\end{aligned}
$$


In view of the assumption made earlier $K(x, t, 0)=0$ and substituting $\bar{\eta}=0$ into to above expression, we can write

$$
A(x, t, \eta) \eta=K(x, t, \eta)-K(x, t, 0)=K(x, t, \eta),
$$

where we used the simplified notation $A(x, t, \eta)=\widetilde{A}(x, t, \eta, 0)$. With the above technique the equation $\mathcal{N}[u]=f$ can be reformulated as

$$
\frac{\partial u}{\partial t}-\operatorname{div}(A(x, t, \nabla u) \nabla u)+r(x, t, u) u=f .
$$

The weak form of the equation can be formulated in a usual way as follows: find $u$ that is $C^{1}$ w.r.t. $t, u(., t) \in H^{1}(\Omega)$ for all $t \in(0, T)$, and $u$ satisfies

$$
\int_{\Omega} \frac{\partial u}{\partial t} v \mathrm{~d} x+\int_{\Omega}(A(x, t, \nabla u) \nabla u \cdot \nabla v+r(x, t, u) u v) \mathrm{d} x=\int_{\Omega} f v \mathrm{~d} x .
$$

$\left(\forall v \in H_{0}^{1}(\Omega), t \in(0, T)\right)$.

The standard semidiscretization of the problem can be carried out as follows. Let $\mathcal{T}_{h}$ be a finite element mesh over the spatial solution domain $\Omega \subset \mathbb{R}^{d}$, where $h$ denotes the usual discretization parameter. We choose basis functions denoted by $\phi_{1}, \ldots, \phi_{\bar{N}}$ such that they satisfy the conditions

$$
\phi_{i}\left(x_{j}\right)=\delta_{i j}, \quad \phi_{i} \geq 0 \quad(i=1, \ldots, \bar{N}), \quad \sum_{i=1}^{\bar{N}} \phi_{i} \equiv 1,
$$

where $\delta_{i j}$ is the Kronecker symbol. Note that the above requirements are fulfilled for the standard linear, bilinear or prismatic finite elements. Let $V_{h}$ and $V_{h}^{0}$ denote the finite element subspaces $V_{h}=\operatorname{span}\left\{\phi_{1}, \ldots, \phi_{\bar{N}}\right\} \subset H^{1}(\Omega), V_{h}^{0}=\operatorname{span}\left\{\phi_{1}, \ldots, \phi_{N}\right\} \subset$ $H_{0}^{1}(\Omega)$, respectively. Then the semidiscrete problem for (15) reads as follows: find a function $u_{h}=u_{h}(x, t), u_{h}(., t) \in V_{h}(t \in(0, T))$ such that

$$
\int_{\Omega} \frac{\partial u_{h}}{\partial t} v_{h} d x+\int_{\Omega}\left(A\left(x, t, \nabla u_{h}\right) \nabla u_{h} \cdot \nabla v_{h}+r\left(x, t, u_{h}\right) u_{h} v_{h}\right) \mathrm{d} x=\int_{\Omega} f v_{h} \mathrm{~d} x
$$

$\left(\forall v_{h} \in V_{h}^{0}, t \in(0, T)\right)$. We do not prescribe now the initial and boundary conditions, these will be included in the studied properties as shown by Definition 2. We seek $u_{h}$ in the form

$$
u_{h}(x, t)=\sum_{i=1}^{\bar{N}} u_{i}^{h}(t) \phi_{i}(x)
$$

Inserting (18) into (17) with $v_{h}=\phi_{i}$ and introducing $u^{h}(t)=\left[u_{1}^{h}(t), \ldots, u_{\bar{N}}^{h}(t)\right]^{T}$, we are led to the following system of ordinary differential equations:

$$
M \frac{\mathrm{d} u^{h}(t)}{\mathrm{d} t}+S^{\left(u^{h}(t)\right)} u^{h}(t)=f^{h}(t)
$$


where

$$
\begin{aligned}
& M=\left[M_{i j}\right]_{N \times \bar{N}}, \quad M_{i j}=\int_{\Omega} \phi_{j}(x) \phi_{i}(x) \mathrm{d} x, \\
& S^{\left(u^{h}(t)\right)}=S_{1}^{\left(u^{h}(t)\right)}+S_{2}^{\left(u^{h}(t)\right)}, \\
& S_{1}^{\left(u^{h}(t)\right)}=\left[\left(S_{1}^{\left(u^{h}(t)\right)}\right)_{i j}\right]_{N \times \bar{N}}, \quad S_{2}^{\left(u^{h}(t)\right)}=\left[\left(S_{2}^{\left(u^{h}(t)\right)}\right)_{i j}\right]_{N \times \bar{N}}, \\
& \left(S_{1}^{\left(u^{h}(t)\right)}\right)_{i j}=\int_{\Omega} A\left(x, t, \nabla u_{h}\right) \nabla \phi_{j} \cdot \nabla \phi_{i} \mathrm{~d} x, \\
& \left(S_{2}^{\left(u^{h}(t)\right)}\right)_{i j}=\int_{\Omega} r\left(x, t, u_{h}\right) \phi_{j} \phi_{i} \mathrm{~d} x, \\
& f^{h}(t)=\left[f_{i}^{h}(t)\right]_{N \times 1}, \quad f_{i}^{h}(t)=\int_{\Omega} f(x, t) \phi_{i}(x) \mathrm{d} x .
\end{aligned}
$$

The function $u^{h}=u^{h}(t)$ is generally called the semidiscrete solution. In order to get a fully discrete numerical scheme, we choose a time-step $\Delta t$ and denote the approximation to $u^{h}(n \Delta t)$ and $f^{h}(n \Delta t)$ by $v^{n}$ and $f^{n}$ (for $n=0,1,2, \ldots, n_{T}$ ), respectively.

To discretize (19) in time, we apply the so-called $\theta$-method with some given parameter $\theta \in(0,1]$. (The case $\theta=0$ is omitted for practical reasons, and it does not have the advantage of explicitness unlike in the case of finite difference methods.) We thus obtain a system of nonlinear algebraic equations

$$
M \frac{v^{n}-v^{n-1}}{\Delta t}+\theta S^{\left(v^{n}\right)} v^{n}+(1-\theta) S^{\left(v^{n-1}\right)} v^{n-1}=f^{(n, \theta)}:=\theta f^{n}+(1-\theta) f^{n-1}
$$

$n=1, \ldots, n_{T}$. Let us introduce the well-defined matrix

$$
P:=\left(\operatorname{diag}\left(\int_{\Omega} \phi_{i}, \ldots, \int_{\Omega} \phi_{N}\right)\right)^{-1} \in \mathbb{R}^{N \times N}
$$

We may multiply the above equality with the matrix $P$ from left: using notation $\tilde{M}:=(1 / \Delta t) P M$,

$$
\tilde{M}\left(v^{n}-v^{n-1}\right)+\theta P S^{\left(v^{n}\right)} v^{n}+(1-\theta) P S^{\left(v^{n-1}\right)} v^{n-1}=P f^{(n, \theta)},
$$

which can be reformulated in the form

$$
X_{1}^{\left(v^{n}\right)} v^{n}-X_{2}^{\left(v^{n-1}\right)} v^{n-1}=P f^{(n, \theta)}
$$

where $X_{1}^{\left(v^{n}\right)}=\tilde{M}+\theta P S^{\left(v^{n}\right)}, X_{2}^{\left(v^{n-1}\right)}=\tilde{M}-(1-\theta) P S^{\left(v^{n-1}\right)}$. Note that the left-hand side of (23) defines a DMO2 for the mesh function $v$, that is,

$$
(D[v])^{n}=\left(\tilde{M}+\theta P S^{\left(v^{n}\right)}\right) v^{n}-\left(\tilde{M}-(1-\theta) P S^{\left(v^{n-1}\right)}\right) v^{n-1},
$$


which is the discrete equivalent of the continuous operator (1). Hence, for the desired qualitative study of the present finite element problem, it is enough to ensure that (24) satisfies the conditions formulated for DMO2s in the previous section.

We formulate some properties of the above matrices.

Lemma 1 Let $z=\left[z_{1}, \ldots, z_{\bar{N}}\right]^{T} \in \mathbb{R}^{\bar{N}}$ be an arbitrary column vector, and let $z_{h}:=$ $\sum_{k=1}^{\bar{N}} z_{k} \phi_{k}$. Then

(i) $S_{1}^{(z)} e=0$.

(ii) $\left(S_{2}^{(z)} z\right)_{i}=\int_{\Omega} q\left(x, t, z_{h}\right) \phi_{i} \mathrm{~d} x, \quad i=1, \ldots, N$.

(iii) $S_{1}^{(z+c e)}=S_{1}^{(z)}$ for any real constant $c$.

(iv) The matrix $M$ is nonnegative and the vector $M e$ is positive.

Proof We will use repeatedly the third condition in (16).

(i) The $i$ th coordinate satisfies

$$
\begin{aligned}
\left(S_{1}^{(z)} e\right)_{i} & =\sum_{j=1}^{\bar{N}}\left(\int_{\Omega} A\left(x, t, \nabla z_{h}\right) \nabla \phi_{j} \cdot \nabla \phi_{i} \mathrm{~d} x\right) \\
& =\int_{\Omega} A\left(x, t, \nabla z_{h}\right) \nabla\left(\sum_{j=1}^{\bar{N}} \phi_{j}\right) \cdot \nabla \phi_{i} \mathrm{~d} x=\int_{\Omega} A\left(x, t, \nabla z_{h}\right) \nabla 1 \cdot \nabla \phi_{i} \mathrm{~d} x=0 .
\end{aligned}
$$

(ii) Applying the reformulation (11),

(iii)

$$
\begin{aligned}
\left(S_{2}^{(z)} z\right)_{i} & =\sum_{j=1}^{\bar{N}}\left(\int_{\Omega} r\left(x, t, z_{h}\right) \phi_{j} \phi_{i} \mathrm{~d} x\right) z_{j} \\
& =\int_{\Omega} r\left(x, t, z_{h}\right) z_{h} \phi_{i} \mathrm{~d} x=\int_{\Omega} q\left(x, t, z_{h}\right) \phi_{i} . \\
\left(S_{1}^{(z+c e)}\right)_{i j} & =\int_{\Omega} A\left(x, t, \nabla\left(\sum_{k=1}^{\bar{N}}\left(z_{k}+c\right) \phi_{k}\right)\right) \nabla \phi_{j} \cdot \nabla \phi_{i} \mathrm{~d} x \\
& =\int_{\Omega} A\left(x, t, \nabla\left(z_{h}+c\right)\right) \nabla \phi_{j} \cdot \nabla \phi_{i} \mathrm{~d} x=\left(S_{1}^{(z)}\right)_{i j} .
\end{aligned}
$$

(iv) The nonnegativity of the matrix $M$ follows from the nonnegativity of the basis functions $\phi_{i}$; further,

$$
(M e)_{i}=\sum_{j=1}^{\bar{N}} \int_{\Omega} \phi_{j} \phi_{i} \mathrm{~d} x=\int_{\Omega}\left(\sum_{j=1}^{\bar{N}} \phi_{j}\right) \phi_{i} \mathrm{~d} x=\int_{\Omega} \phi_{i} \mathrm{~d} x>0 .
$$

Thus the proof of the theorem is complete. 


\subsection{Implication of discrete maximum/minimum principles}

Now we are ready to give a sufficient condition for the relations involving discrete maximum/minimum principles. Based on the previous results, this problem can be reduced to Theorem 6, i.e. to ensuring conditions (W) or (S) for the weak or strong forms of the principles, respectively.

Theorem 10 If the function $\xi \mapsto q(x, t, \xi)$ is nondecreasing, then the DNPP (or $D N N P$ ) property implies the discrete weak maximum principles DWMP and DWBMP (or discrete weak minimum principles $D W m P$ and $D W B m P$, respectively) for the DMO2 (24).

Proof In order to apply Theorem 6, we need to show that the condition $(W)$ is satisfied. Consider an arbitrary vector $w_{1} \in \mathbb{R}^{\bar{N}}$ and a positive constant $a$. Applying Lemma 1 (i)-(iii), using the nonnegativity of the basis functions and that the function $\xi \mapsto$ $q(x, t, \xi)$ is nondecreasing, we have

$$
S_{1}^{\left(w_{1}-a e\right)}\left(w_{1}-a e\right)=S_{1}^{\left(w_{1}\right)} w_{1}, \quad S_{2}^{\left(w_{1}-a e\right)}\left(w_{1}-a e\right) \leq S_{2}^{\left(w_{1}\right)} w_{1},
$$

hence $S^{\left(w_{1}-a e\right)}\left(w_{1}-a e\right) \leq S^{\left(w_{1}\right)} w_{1}$. In order to show property $(W)$, let us fix the arbitrary vectors $w_{1}$ and $w_{2}$ and the nonnegative numbers $a \geq b \geq 0$. Using also the properties (i) and (iii)-(iv) in Lemma 1 the required estimation can be carried out as follows:

$$
\begin{aligned}
& J\left(w_{1}-a e, w_{2}-b e\right)=X_{1}^{\left(w_{1}-a e\right)}\left(w_{1}-a e\right)-X_{2}^{\left(w_{2}-b e\right)}\left(w_{2}-b e\right) \\
& \quad=\left(\tilde{M}+\theta P S^{\left(w_{1}-a e\right)}\right)\left(w_{1}-a e\right)-\left(\tilde{M}-(1-\theta) P S^{\left(w_{2}-b e\right)}\right)\left(w_{2}-b e\right) \\
& \leq \tilde{M}\left(w_{1}-a e\right)+\theta P S^{\left(w_{1}\right)} w_{1}-\tilde{M}\left(w_{2}-b e\right)+(1-\theta) P S^{\left(w_{2}\right)} w_{2} \\
& \quad=X_{1}^{\left(w_{1}\right)} w_{1}-X_{2}^{\left(w_{2}\right)} w_{2}-\tilde{M}(a-b) e=X_{1}^{\left(w_{1}\right)} w_{1}-X_{2}^{\left(w_{2}\right)} w_{2}-\frac{a-b}{\Delta t} P M e \\
& \quad=X_{1}^{\left(w_{1}\right)} w_{1}-X_{2}^{\left(w_{2}\right)} w_{2}-\frac{a-b}{\Delta t} e_{0}=J\left(w_{1}, w_{2}\right)-\frac{a-b}{\Delta t} e_{0},
\end{aligned}
$$

where we used the equality $P M e=e_{0}$.

Theorem 11 If $q(x, t, \xi) \equiv 0$, then the DNPP (or DNNP) property implies the discrete strong maximum principles DMP and DBMP (or discrete strong minimum principles DmP and WBmP, respectively) for the DMO2 (24).

Proof Now we need to show the condition $(S)$. This goes in the same way as the proof Theorem 10 such that the inequalities therein are replaced by equalities.

Remark 5 Note that the conditions of the previous two theorems are the same as those used to guarantee the same implications in the continuous case (see Fig. 1). That is, there is no additional condition in the finite element case.

Corollary 1 In the case $q(x, t, \xi)=0$, if the DMO2 (24) possesses the DNPP property then it fulfills all the discrete maximum principles as well, and if the DMO2 (24) possesses the DNNP property then it fulfills all the minimum principles as well. 


\subsection{Implication of discrete maximum norm contractivity}

Now we give conditions for the relation involving the DMNC property. This relies on a proper linearization of the nonlinear matrix function $S^{(.)}$between given $\bar{z}$ and $\tilde{z}$, defined as follows:

$$
\left(S^{(\bar{z}, \tilde{z})}\right)_{i j}:=\int_{\Omega} \tilde{A}\left(x, t, \nabla \bar{z}_{h}, \nabla \tilde{z}_{h}\right) \nabla \phi_{j} \cdot \nabla \phi_{i} \mathrm{~d} x+\int_{\Omega} \tilde{r}\left(x, t, \bar{z}_{h}, \tilde{z}_{h}\right) \phi_{j} \phi_{i} \mathrm{~d} x .
$$

Theorem 12 Let us suppose that

(I) the function $\xi \mapsto q(x, t, \xi)$ is nondecreasing, and

(L) for any two fixed discrete mesh functions $\bar{w}$ and $\tilde{w}$, the mesh operators defined as

$$
\left(L_{\bar{w}, \tilde{w}}[v]\right)^{n}=\left(\tilde{M}+\theta P S^{\left(\bar{w}^{n}, \tilde{w}^{n}\right)}\right) v^{n}-\left(\tilde{M}-(1-\theta) P S^{\left(\bar{w}^{n-1}, \tilde{w}^{n-1}\right)}\right) v^{n-1}
$$

$\left(n=1, \ldots, n_{T}\right)$, where $S^{(. .)}$stands for the linearized matrix function defined above, possess both the DNNP and DNPP properties.

Then the DMO2 (23) possesses the DMNC property.

Proof We have to show that under the assumptions of the theorem the assumptions $\left(\mathrm{W}_{=}\right)$and $(\mathrm{L})$ in Theorem 9 are satisfied. Condition $\left(\mathrm{W}_{=}\right)$can be shown in a similar way as condition (W) in Theorem 10 , using the fact that $a=b$.

It is left to show that the condition $(\mathrm{L})$ of this theorem implies the condition $(\mathrm{L})$ in Theorem 9. Thus we have to show the equality $L_{\bar{w}, \tilde{w}}[\bar{w}-\tilde{w}]=D[\bar{w}]-D[\tilde{w}]$, where $D$ is the DMO2 defined in (24). In view of equalities (10) and (12), for any two vectors $\bar{z}, \tilde{z} \in \mathbb{R}^{\bar{N}}$ we have

$$
\begin{aligned}
& \left(S^{(\bar{z}, \tilde{z})}(\bar{z}-\tilde{z})\right)_{i}=\sum_{j=1}^{\bar{N}}\left(S^{(\bar{z}, \tilde{z})}\right)_{i j}\left(\bar{z}_{j}-\tilde{z}_{j}\right) \\
& =\int_{\Omega} \tilde{A}\left(x, t, \nabla \bar{z}_{h}, \nabla \tilde{z}_{h}\right)\left(\nabla \bar{z}_{h}-\nabla \tilde{z}_{h}\right) \cdot \nabla \phi_{i} \mathrm{~d} x+\int_{\Omega} \tilde{r}\left(x, t, \bar{z}_{h}, \tilde{z}_{h}\right)\left(\bar{z}_{h}-\tilde{z}_{h}\right) \phi_{i} \mathrm{~d} x \\
& =\int_{\Omega}\left(K\left(x, t, \nabla \bar{z}_{h}\right)-K\left(x, t, \nabla \tilde{z}_{h}\right)\right) \cdot \nabla \phi_{i} \mathrm{~d} x+\int_{\Omega}\left(q\left(x, t, \bar{z}_{h}\right)-q\left(x, t, \tilde{z}_{h}\right)\right) \phi_{i} \mathrm{~d} x \\
& =\int_{\Omega}\left(A\left(x, t, \nabla \bar{z}_{h}\right) \nabla \bar{z}_{h}-A\left(x, t, \nabla \tilde{z}_{h}\right) \nabla \tilde{z}_{h}\right) \cdot \nabla \phi_{i} \mathrm{~d} x \\
& \quad+\int_{\Omega}\left(r\left(x, t, \bar{z}_{h}\right) \bar{z}_{h}-r\left(x, t, \tilde{z}_{h}\right) \tilde{z}_{h}\right) \phi_{i} \mathrm{~d} x=\left(S^{(\bar{z})} \bar{z}-S^{(\tilde{z})} \tilde{z}\right)_{i} .
\end{aligned}
$$

Thus we obtain that

$$
\begin{aligned}
& \left(L_{\bar{w}, \tilde{w}}[\bar{w}-\tilde{w}]\right)^{n} \\
& \quad=\left(\tilde{M}+\theta P S^{\left(\bar{w}^{n}, \tilde{w}^{n}\right)}\right)\left(\bar{w}^{n}-\tilde{w}^{n}\right)-\left(\tilde{M}-(1-\theta) P S^{\left(\bar{w}^{n-1}, \tilde{w}^{n-1}\right)}\right)\left(\bar{w}^{n-1}-\tilde{w}^{n-1}\right)
\end{aligned}
$$




$$
\begin{aligned}
= & \tilde{M}\left(\bar{w}^{n}-\tilde{w}^{n}\right)+\theta P\left(S^{\left(\bar{w}^{n}\right)} \bar{w}^{n}-S^{\left(\tilde{w}^{n}\right)} \tilde{w}^{n}\right) \\
& -\left(\tilde{M}\left(\bar{w}^{n-1}-\tilde{w}^{n-1}\right)-(1-\theta) P\left(S^{\left(\bar{w}^{n-1}\right)} \bar{w}^{n-1}-S^{\left(\tilde{w}^{n-1}\right)} \tilde{w}^{n-1}\right)\right) \\
= & \left(\tilde{M}+\theta P S^{\left(\bar{w}^{n}\right)}\right) \bar{w}^{n}-\left(\tilde{M}-(1-\theta) P S^{\left(\bar{w}^{n-1}\right)}\right) \bar{w}^{n-1} \\
& -\left(\left(\tilde{M}+\theta P S^{\left(\tilde{w}^{n}\right)}\right) \tilde{w}^{n}-\left(\tilde{M}-(1-\theta) P S^{\left(\tilde{w}^{n-1}\right)}\right) \tilde{w}^{n-1}\right)=(D[\bar{w}]-D[\tilde{w}])^{n}
\end{aligned}
$$

$\left(n=1, \ldots, n_{T}\right)$, which completes the proof.

\subsection{Ensuring the DNNP-DNPP properties}

In the above we have seen that under certain conditions the DNNP or DNPP implies the other studied properties for the DMO2 (24). Now we give conditions to ensure that DNNP and DNPP hold themselves. Altogether, in this way we can also ensure the validity of all the qualitative properties for the finite element mesh operator.

\subsubsection{The general case}

Definition 3 A finite element mesh $\mathcal{T}_{h}$ is called strictly non-degenerate with respect to the basis functions $\phi_{1}, \ldots, \phi_{\bar{N}}$ and the coefficient functions $K$ and $q$ of the operator (1) (or shortly strictly non-degenerate), if the following condition holds. For any $i=$ $1, \ldots, N, j=1, \ldots, \bar{N}$ and $i \neq j$, whenever the basis functions $\phi_{i}$ and $\phi_{j}$ have overlapping support, we have

$$
S_{i j}^{(z)}=\int_{\Omega}\left(A\left(x, t, \nabla z_{h}\right) \nabla \phi_{j} \cdot \nabla \phi_{i}+r\left(x, t, z_{h}\right) \phi_{j} \phi_{i}\right) \mathrm{d} x<0
$$

for all vectors $z \in \mathbb{R}^{\bar{N}}$, where $A$ and $r$ are the functions defined in (11) and (13) with the coefficient functions $K$ and $q$, respectively, and where we denote $z_{h}=\sum_{k=1}^{\bar{N}} z_{k} \phi_{k}$.

Remark 6 When $K(x, t, \nabla u)=\nabla u$ and $q=0$ then for piecewise linear elements on triangular meshes the strict non-degenerateness means the well-known acute angle condition: all angles of the triangles in the triangulation must be less then $\pi / 2$. For bilinear elements on rectangular mesh the property means the strict non-narrowness of the rectangles. Such geometric conditions can be used to ensure (27) in the general case as well, as will be illustrated by Theorem 14 below.

Now we are ready to give sufficient conditions for the DNNP and DNPP properties of the DMO2 (24).

Theorem 13 Let us suppose that

(i) $\xi \mapsto q(x, t, \xi)$ is nondecreasing;

(ii) we use a strictly non-degenerate mesh in the construction of the finite element solution.

Then there exist positive numbers $\Delta t_{\min }$ and $\Delta t_{\max }$ such that if $\Delta t_{\min } \leq \Delta t \leq \Delta t_{\max }$ then the DMO2 (24) possesses the property DNPP and DNNP. 
Proof We apply the conditions listed in Theorem 8 . Let $z \in \mathbb{R}^{\bar{N}}$ be an arbitrary vector.

We first check the nonnegativity of the matrix $X_{2}^{(z)}$. In view of the nonnegativity of the matrices $P$ and $M$ and the strict non-degeneracy of the mesh, the off-diagonal elements of $X_{2}^{(z)}=(1 / \Delta t) P M-(1-\theta) P S^{(z)}$ are trivially nonnegative. The nonnegativity of the diagonal elements yields the condition

$$
\frac{1}{\int_{\Omega} \phi_{i} \mathrm{~d} x}\left(\frac{1}{\Delta t} \int_{\Omega} \phi_{i}^{2} \mathrm{~d} x-(1-\theta) \int_{\Omega}\left(A\left(x, t, \nabla z_{h}\right) \nabla \phi_{i} \cdot \nabla \phi_{i}+r\left(x, t, z_{h}\right) \phi_{i}^{2}\right) \mathrm{d} x\right) \geq 0
$$

$(i=1, \ldots, N)$. This condition is trivially satisfied if $\Delta t$ is sufficiently small, say $\Delta t \leq \Delta t_{\max }$ for some appropriate positive value $\Delta t_{\max }$.

Now we check the non-positivity of the off-diagonal elements of

$$
X_{1}^{(z)}=(1 / \Delta t) P M+\theta P S^{(z)} .
$$

If the basis functions $\phi_{i}$ and $\phi_{j}$ have disjoint support, then clearly $\left(X_{1}^{(z)}\right)_{i j}=0$. If $\phi_{i}$ and $\phi_{j}$ have overlapping support, then using the condition $\theta>0$, the strict non-degeneracy of the mesh and the positivity of the diagonal elements of $P$, the offdiagonal elements of the matrix $\theta P S^{(z)}$ are negative. Thus, in view of the nonnegativity of $P M$, if $\Delta t$ is sufficiently large, say $\Delta t \geq \Delta t_{\min }$ for some appropriate positive value $\Delta t_{\min }$, then the off-diagonal elements of $X_{1}^{(z)}$ are nonpositive. This condition guarantees the condition $X_{1 \partial}^{(z)} \leq 0$.

Now we show that the matrix $X_{10}^{(0)}$ is nonsingular and its inverse is nonnegative. We will see that this condition does not requires any additional assumption. We do this with the usual $M$-matrix technique. In the previous paragraph, we showed that if $\Delta t \geq \Delta t_{\min }$ then the off-diagonal of $X_{1}^{(z)}$ is nonpositive. In order to show that the matrix $X_{10}^{(z)}$ is an $M$-matrix (that is it is regular and its inverse is nonnegative) it is enough to show that the product $X_{1}^{(z)} e$ is positive (hence in view of the nonpositivity of $X_{12}^{(z)}$ the vector $X_{10}^{(z)} e_{0}$ is also positive). Here we have

$$
X_{1}^{(z)} e=\frac{1}{\Delta t} e_{0}+\theta P S_{2}^{(z)} e=\frac{1}{\Delta t} e_{0}+\theta\left[\frac{\int_{\Omega} r\left(x, t, z_{h}\right) \phi_{i} \mathrm{~d} x}{\int_{\Omega} \phi_{i} \mathrm{~d} x}\right]_{i=1, \ldots, N},
$$

where we used property (i) in Lemma 1 . Because $q$ is nondecreasing in its third argument, the function $r$ is nonnegative. Thus the above vector is positive, and this was what we wanted to show.

Remark 7 (On the strict non-degenerateness property) The strict non-degenerateness of the finite element mesh is clearly a crucial property for the final results, and it can be ensured with various sufficient conditions. This has been studied in detail in our papers $[9,10]$, depending on the structural conditions of the PDE problem. Here we show a case where a brief set of sufficient conditions can be given. Namely, strict non-degenerateness can be ensured with the following conditions: assume that 
(i) the coefficient $q$ has a bounded derivative w.r.t. $\xi$, i.e. there exists a constant $\alpha>0$ such that

$$
0 \leq \frac{\partial q(x, t, \xi)}{\partial \xi} \leq \alpha
$$

(ii) the finite element meshes are regular in the sense that there exists a positive number $C_{m}$ such that

$$
\operatorname{meas}_{d}\left(\operatorname{supp}\left(\phi_{i}\right)\right) \leq C_{m} h^{d}
$$

$(\forall i=1, \ldots, \bar{N})$, where meas $_{d}$ denotes the $d$-dimensional measure and $\operatorname{supp}\left(\phi_{i}\right)$ is the support of $\phi_{i}$;

(iii) there exists a positive constant $\mu>0$, depending on the coefficient (13), such that

$$
\int_{\Omega} A(x, t, \eta) \nabla \phi_{i} \cdot \nabla \phi_{j} \mathrm{~d} x \leq-\mu h^{d-2} \quad(\forall i \neq j) ;
$$

whenever the basis functions $\phi_{i}$ and $\phi_{j}$ have overlapping support;

(iv) the mesh is fine enough, namely, it satisfies the condition

$$
h<h_{0}:=\sqrt{\frac{\mu}{\alpha C_{m}}} .
$$

Under the conditions (i)-(iv) the finite element mesh is strictly non-degenerate (see Definition 3). Indeed, for the indices $i \neq j$ and an arbitrary vector $z \in \mathbb{R}^{\bar{N}}$, we have the estimation

$$
\begin{aligned}
S_{i j}^{(z)} & =\int_{\Omega}\left(A\left(x, t, \nabla z_{h}\right) \nabla \phi_{j} \cdot \nabla \phi_{i}+r\left(x, t, z_{h}\right) \phi_{j} \phi_{i}\right) \mathrm{d} x \leq-\mu h^{d-2}+\alpha C_{m} h^{d} \\
& =h^{d}\left(\alpha C_{m}-\mu / h^{2}\right)<h^{d}\left(\alpha C_{m}-\mu / h_{0}^{2}\right)=0
\end{aligned}
$$

where we also used the properties (16) and the fact that $r$ inherits the bound $\alpha$ from $\frac{\partial q}{\partial \xi}$.

Remark 8 (i) The magnitude of $\Delta t_{\min }$ and $\Delta t_{\max }$ in Theorem 13 has been considered in a similar situation in the study of DWMP in [10]. A study of the expressions where they appear in Theorem 13 shows that both bounds give

$$
\Delta t=O\left(h^{2}\right)
$$

as $h \rightarrow 0$ for the $\theta$-method for $\theta<1$, and especially, as seen immediately from (28), there is no upper bound in the implicit case $\theta=1$.

To sum up, based on the above, we can say that the discrete weak maximum/minimum principles hold if we use a fine enough regular strictly nondegenerate space mesh together with a time division satisfying $\Delta t=O\left(h^{2}\right)$.

(ii) The discrete maximum norm contractivity can be ensured in a similar way as above, since the linearized mesh operator (25)-(26) contains coefficients $\tilde{A}$ and $\tilde{r}$ in analogy with the coefficients $A$ and $r$ in (27); in particular, the monotonicity of $q$ implies $\tilde{r} \geq 0$. That is, a fine enough regular strictly non-degenerate space mesh, together with a time division satisfying (34), provides the DMNC as well. 


\subsubsection{A special case: scalar diffusion coefficient}

Based on the above observations, one can easily treat a relevant situation when the diffusion coefficient is a scalar function, which is the case in most practical situations. For such problems, (14) becomes

$$
\frac{\partial u}{\partial t}-\operatorname{div}(a(x, t, \nabla u) \nabla u)+r(x, t, u) u=f
$$

(in this case $A(x, t, \eta)=a(x, t, \eta) I$, where $I$ is the identity matrix), where $a$ : $\Omega \times(0, T) \times \mathbb{R}^{d} \rightarrow \mathbb{R}$ is some given continuous scalar function, assumed to be bounded from both sides: $m \leq a(x, t, \eta) \leq M\left(\forall(x, t, \eta) \in \Omega \times(0, T) \times \mathbb{R}^{d}\right)$ for some constants $M \geq m>0$. The following theorem gives sufficient conditions that can be checked a priori, i.e. its assumptions contain computable constants (from the data or the generated mesh).

Theorem 14 Let $\Omega \subset \mathbb{R}^{d}$ for $d=2$ or 3 , and suppose that

(i) the coefficient $q$ in (1) satisfies (29) for some $\alpha>0$;

(ii) we consider piecewise linear finite elements on a regular and strictly acute simplicial mesh, i.e. all diameters $h_{S}$ of the simplices $S$ satisfy $\varrho h \leq h_{S} \leq h$ for some constant $0<\varrho \leq 1$ independent of the mesh, and all angles $\gamma$ (in $3 D$ including also the face angles) are bounded as $0<\gamma_{0} \leq \gamma \leq \gamma_{1}<\pi / 2$;

(iii) letting $h_{0}:=\sqrt{m \kappa_{d} \cos \gamma_{1} \sin ^{2 d-3} \gamma_{0} \varrho^{d-2} /\left(\alpha 2^{d}\right)}$, where $\kappa_{2}:=1 / 8$ and $\kappa_{3}:=$ $1 / 128$, the mesh parameter $h$ satisfies $h<h_{0}$.

Then there exist positive numbers $\Delta t_{\min }$ and $\Delta t_{\max }$ such that if $\Delta t_{\min } \leq \Delta t \leq \Delta t_{\max }$ then the DMO2 (24) possesses the property DNPP and DNNP.

Proof We apply Theorem 13. First, the function $q$ is nondecreasing by (29). We must now verify that the mesh is strictly non-degenerate. This is based on Remark 7, so we check conditions (29)-(32). First, (29) holds by assumption. Now, consider basis functions $\phi_{i}$ and $\phi_{j}$ that have overlapping support, and consider a simplex $S \subset \operatorname{supp} \phi_{i} \cap \operatorname{supp} \phi_{j}$ with diameter $h_{S}$. Then the strictly acute angle condition implies

$$
\nabla \phi_{i} \cdot \nabla \phi_{j} \leq-\sigma h_{S}^{-2} \quad \text { on } S
$$

where $\sigma:=\cos \gamma_{1}>0$ (see e.g. [14]). Then $\operatorname{meas}_{d}(S) \geq \kappa_{d} \sin ^{2 d-3} \gamma_{0} h_{S}^{d}$ with $\kappa_{d}$ from assumption (iii), see [2]. Hence

$$
\begin{aligned}
& \int_{\Omega} a(x, t, \eta) \nabla \phi_{i} \cdot \nabla \phi_{j} \mathrm{~d} x \leq m \int_{S} \nabla \phi_{i} \cdot \nabla \phi_{j} \mathrm{~d} x \\
& \quad \leq-m \sigma h_{S}^{-2} \operatorname{meas}_{d}(S) \leq-m \sigma \kappa_{d} \sin ^{2 d-3} \gamma_{0} h_{S}^{d-2} \leq-\mu h^{d-2}
\end{aligned}
$$

with $\mu:=m \sigma \kappa_{d} \sin ^{2 d-3} \gamma_{0} \varrho^{d-2}$, i.e. (31) holds. Finally we note that any $\operatorname{supp}\left(\phi_{i}\right)$ is contained in a square (cube) with edge $2 h$, hence (30) holds with $C_{m}=2^{d}$. Thus we obtain that 


$$
h<h_{0}:=\sqrt{\frac{m \kappa_{d} \cos \gamma_{1} \sin ^{2 d-3} \gamma_{0} \varrho^{d-2}}{\alpha 2^{d}}}=\sqrt{\frac{m \sigma \kappa_{d} \sin ^{2 d-3} \gamma_{0} \varrho^{d-2}}{\alpha 2^{d}}}=\sqrt{\frac{\mu}{\alpha C_{m}}},
$$

i.e. (32) also holds.

Remark 9 The strictly acute angle condition can be relaxed so that estimates like (36) can still be executed, see [10]: some obtuse interior angles may occur in the simplices of the meshes, or alternatively, nonobtuseness suffices if one requires strict acuteness on a proper (asymptotically non-vanishing) subpart of each intersection of supports.

Remark 10 One can give explicit bounds on $\Delta t_{\min }$ and $\Delta t_{\max }$ so that Theorem 14 holds, depending on the dimension and coefficients of the problem. This can be illustrated in the 2D case for our model problem (35) as follows. In view of the proof of Theorem 13, the upper bound on $\Delta t$ comes from the condition

$$
\frac{1}{\Delta t} \int_{\Omega} \phi_{i}^{2} \mathrm{~d} x-(1-\theta) \int_{\Omega}\left(a\left(x, t, \nabla z_{h}\right)\left|\nabla \phi_{i}\right|^{2}+r\left(x, t, z_{h}\right) \phi_{i}^{2}\right) \mathrm{d} x \geq 0
$$

as a consequence of (28), and similarly, the lower bound comes from the condition

$$
\frac{1}{\Delta t} \int_{\Omega} \phi_{i} \phi_{j} \mathrm{~d} x+\theta \int_{\Omega}\left(a\left(x, t, \nabla z_{h}\right) \nabla \phi_{i} \cdot \nabla \phi_{j}+r\left(x, t, z_{h}\right) \phi_{i} \phi_{j}\right) \mathrm{d} x \leq 0 .
$$

Clearly, it suffices to have the above estimates on each triangle $T$ for basis functions $\phi_{i}$ and $\phi_{j}$ with overlapping supports. Using the assumed bounds $m \leq a \leq M$ and the relation $0 \leq r \leq \alpha$ (which follows from conditions (11) and (29) and from the Newton-Leibniz rule), some rearrangement and taking maxima yields that a sufficient condition for the desired two estimates is

$$
(1-\theta)\left(M \max _{i} \frac{\int_{T}\left|\nabla \phi_{i}\right|^{2}}{\int_{T} \phi_{i}^{2}}+\alpha\right) \leq \frac{1}{\Delta t} \leq \theta\left(m \min _{i, j} \frac{-\int_{T} \nabla \phi_{i} \cdot \nabla \phi_{j}}{\int_{T} \phi_{i} \phi_{j}}-\alpha\right) .
$$

For the 1.h.s., we have from [11, p. 277] that $\int_{T}\left|\nabla \phi_{i}\right|^{2}=\frac{1}{2}\left(\cot \beta_{i 1}+\cot \beta_{i 2}\right)$ and $\int_{T} \phi_{i}^{2}=|T| / 6$, where $\beta_{i 1}$ and $\beta_{i 2}$ are the angles opposite to the $i$ th node and $|T|$ denotes the area of the triangle $T$. Now let us use condition (ii) of Theorem 14: first, all angles are bounded below by $\gamma_{0}$, hence $\int_{T}\left|\nabla \phi_{i}\right|^{2} \leq \cot \gamma_{0} \leq 1 / \sin \gamma_{0}$, further, all edges are bounded below by $\varrho$, hence $|T| \geq \frac{1}{2}(\varrho h)^{2} \sin \gamma_{0}$. For the r.h.s., the assumptions imply $-\int_{T} \nabla \phi_{i} \cdot \nabla \phi_{j} \geq \cos \gamma_{1} h^{-2}|T|$, further, using [11, p. 277] again, we have $\int_{T} \phi_{i} \phi_{j}=|T| / 12$. Altogether, we have

$$
\max _{i} \frac{\int_{T}\left|\nabla \phi_{i}\right|^{2}}{\int_{T} \phi_{i}^{2}} \leq \frac{12}{\left(\varrho h \sin \gamma_{0}\right)^{2}} \text { and } \min _{i, j} \frac{-\int_{T} \nabla \phi_{i} \cdot \nabla \phi_{j}}{\int_{T} \phi_{i} \phi_{j}} \geq \frac{12 \cos \gamma_{1}}{h^{2}}
$$


Setting the above into (39), we obtain that the condition

$$
(1-\theta)\left(\frac{12 M}{\left(\varrho \sin \gamma_{0}\right)^{2}} \frac{1}{h^{2}}+\alpha\right) \leq \frac{1}{\Delta t} \leq \theta\left(\frac{12 m \cos \gamma_{1}}{h^{2}}-\alpha\right)
$$

is sufficient to ensure that Theorem 14 holds. Altogether, the two sides of (40) are computable bounds for $1 / \Delta t_{\max }$ and $1 / \Delta t_{\min }$ if $h<\sqrt{12 m \cos \gamma_{1} / \alpha}$, which are in accordance with the property $\Delta t=O\left(h^{2}\right)$ as $h \rightarrow 0$. Moreover, since the 1.h.s. of (40) vanishes for $\theta=1$, we also see that the requirement $\Delta t_{\min } \leq \Delta t_{\max }$ can be satisfied by choosing $\theta$ sufficiently close to 1 (the bound on $\theta$ can be calculated readily from (40)), in particular, this always holds for the fully implicit method.

In the more general case than (29), the nonlinearity $q$ may grow superlinearly with some power order:

$$
0 \leq \frac{\partial q(x, t, \xi)}{\partial \xi} \leq \alpha+\beta|\xi|^{p-2}
$$

where $p>2$ is an exponent for which a Sobolev embedding of $H^{1}(\Omega)$ into $L^{p}(\Omega)$ holds. If $\left\|u^{h}\right\|_{L^{p}}$ is bounded for the discrete solutions, then $S_{i j}^{\left(u^{h}\right)}$ can be estimated similarly to (33), but now one obtains an extra term $\beta h^{2 d / p}$ from Hölder's inequality: $-\mu h^{d-2}+\alpha C_{m} h^{d}+\beta h^{2 d / p}$, where the condition $p>2$ yields $2 d / p<d$, and hence the expression still remains negative for small $h$. In this way the mesh is still strictly non-degenerate. The details of such derivations, including the boundedness of $\left\|u^{h}\right\|_{L^{p}}$, can be found in [10].

Typical situations for problems (35) are reaction-diffusion equations

$$
\frac{\partial u}{\partial t}-\operatorname{div}(a(x, t) \nabla u)+q(x, u)=f,
$$

where $a(x, t)$ is a positive bounded diffusion coefficient. For nonlinearities satisfying (29) or (41), respectively, we may mention the Michaelis-Menten reaction $q(x, u)=$ $u /(\varepsilon(u+\kappa))$ in enzyme kynetics and autocatalytic chemical reactions $q(x, u)=u^{\sigma}$ for some $\sigma>1$, see $[4,18]$.

Corollary 2 Under the conditions of Theorem 14, all the discrete weak maximum/minimum principles DWMP, DWBMP, DWmP and DWBmP also hold for the finite element DMO2 (24).

Proof This readily follows from Theorem 10, since assumption (i) of Theorem 14 shows that $\xi \mapsto q(x, t, \xi)$ is nondecreasing.

\subsection{Summary of the results}

The results of the above theorems for the finite element DMO2 (24) are summarized in an implication diagram in Fig. 3. 
Properties of the finite element DMO2 (28)

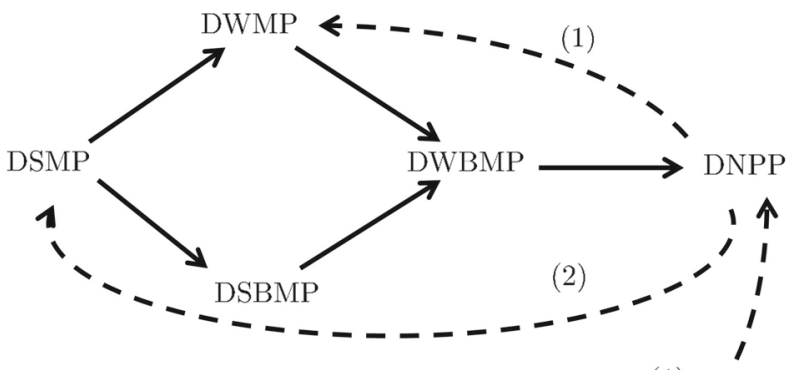

DMNC

N.

1

1

I

(1) $\mathbf{I}$

(1): $\quad \xi \mapsto q(x, t, \xi)$ is nondecreasing

(2): $q(x, t, \xi)=0$

(1)

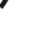

I
(n)

-

The mesh is strictly non-degenerate and

$$
c_{\min } h^{2} \leq \Delta t \leq c_{\max } h^{2}
$$

The specially defined linearized operator in (30) possesses both the DNNP and DNPP properties

Fig. 3 Connections between the qualitative properties of the discrete nonlinear two-level mesh operator (24) that was constructed with the finite element discretization method

\section{Conclusions}

In this paper we have investigated the connections between the maximum/minimum principles, the nonnegativity and nonpositivity preservation and maximum norm contractivity properties of nonlinear discrete mesh operators. First we have revealed the relations between the properties of general mesh operators. We have summarized the implications in Fig. 2. Then we have formulated the same conditions to a special case: to the two-level nonlinear discrete mesh operators. In Theorem 6, we have obtained that the maximum/minimum principles generally imply the nonnegativity and nonpositivity preservation properties but the implications in the opposite direction are valid only under some supplementary assumptions. The discrete maximum norm contractivity property has been guaranteed in Theorem 9. Since we saw that the cornerstones of the implications are the nonnegativity and nonpositivity preservation properties, we turned to the validation of these properties in Theorems 7 and 8. As an application, which is important and interesting also on its own, we have considered the finite element solution of a nonlinear equation. We have shown that beyond the conditions that were required also in the continuous case, the nonnegativity/nonpositivity can be guaranteed by choosing a strictly non-degenerate spatial mesh and an appropriate time step. The implications are summarized in Fig. 3. 
Acknowledgements Open access funding provided by Budapest University of Technology and Economics (BME). The research reported in this paper has been supported by the National Research, Development and Innovation Fund (TUDFO/51757/2019-ITM, Thematic Excellence Program). This research was carried out in the ELTE Institutional Excellence Program (1783-3/2018/FEKUTSRAT) supported by the Hungarian Ministry of Human Capacities, and further, it was supported by the Hungarian Scientific Research Fund OTKA, Nos. K112157 and SNN125119.

Open Access This article is distributed under the terms of the Creative Commons Attribution 4.0 International License (http://creativecommons.org/licenses/by/4.0/), which permits unrestricted use, distribution, and reproduction in any medium, provided you give appropriate credit to the original author(s) and the source, provide a link to the Creative Commons license, and indicate if changes were made.

\section{References}

1. Borisov, V.S., Sorek, S.: On the monotonicity of difference schemes for computational physics. SIAM J. Sci. Comput. 25, 1557-1584 (2004)

2. Brandts, J., Korotov, S., Kř́̌žek, M.: On the equivalence of regularity criteria for triangular and tetrahedral finite element partitions. Comput. Math. Appl. 55(10), 2227-2233 (2008)

3. Csóka, J., Faragó, I., Horváth, R., Karátson, J., Korotov, S.: Qualitative properties of nonlinear parabolic operators II: the case of PDE systems. J. Math. Anal. Appl. 468(1), 64-86 (2018)

4. Díaz, J.I.: Applications of symmetric rearrangement to certain nonlinear elliptic equations with a free boundary. In: Nonlinear Differential Equations (Granada, 1984). Research Notes Mathematics, vol. 132, pp. 155-181. Pitman, Boston (1985)

5. Faragó, I., Horváth, R.: Discrete maximum principle and adequate discretizations of linear parabolic problems. SIAM Sci. Comput. 28, 2313-2336 (2006)

6. Faragó, I., Horváth, R.: Continuous and discrete parabolic operators and their qualitative properties. IMA J. Numer. Anal. 29, 606-631 (2009)

7. Faragó, I., Horváth, R., Korotov, S.: Discrete maximum principles for FE solutions of nonstationary diffusion-reaction problems with mixed boundary conditions. Numer. Methods Partial Differ. Equ. 27(3), 702-720 (2011)

8. Faragó, I., Horváth, R., Karátson, J., Korotov, S.: Qualitative properties of nonlinear parabolic operators. J. Math. Anal. Appl. 448(1), 473-497 (2017)

9. Faragó, I., Karátson, J., Korotov, S.: Discrete maximum principles for the FEM solution of some nonlinear parabolic problems. ETNA Electron. Trans. Numer. Anal. 36, 149-167 (2009)

10. Faragó, I., Karátson, J., Korotov, S.: Discrete maximum principles for nonlinear parabolic PDE systems. IMA J. Numer. Anal. 32(4), 1541-1573 (2012)

11. Hannukainen, A., Korotov, S., Vejchodský, T.: Discrete maximum principle for FE solutions of the diffusion-reaction problem on prismatic meshes. J. Comput. Appl. Math. 226, 275-287 (2009)

12. Hansen, E., Kramer, F., Ostermann, A.: A second-order positivity preserving scheme for semilinear parabolic problems. Appl. Numer. Math. 62(10), 1428-1435 (2012)

13. Jerome, J.W.: A trapping principle and convergence result for finite element approximate solutions of steady reaction/diffusion systems. Numer. Math. 109(1), 121-142 (2008)

14. Karátson, J., Korotov, S.: Discrete maximum principles for finite element solutions of nonlinear elliptic problems with mixed boundary conditions. Numer. Math. 99, 669-698 (2005)

15. Koleva, M.N., Vulkov, L.G.: Positivity preserving numerical method for optimal portfolio in a power utility two-dimensional regime-switching model. In: Numerical Methods and Applications. Lecture Notes in Computer Sciences, vol. 11189, pp. 424-432 (2018)

16. Kraaijevanger, J.F.B.M.: Maximum norm contractivity of discretization schemes for the heat equation. Appl. Numer. Math. 9, 475-492 (1992)

17. Krŕřžek, M., Liu, L.: On the maximum and comparison principles for a steady-state nonlinear heat conduction problem. ZAMM Z. Angew. Math. Mech. 83, 559-563 (2003)

18. Lyons, M.G., et al.: Reaction-diffusion with Michaelis-Menten kinetics in electroactive polymer films. Analyst 12, 715-731 (1996)

19. Li, H., Wang, M.: Properties of positive solutions to a nonlinear parabolic problem. Sci. China Ser. A: Math. 50(4), 590-608 (2007) 
20. Li, X., Huang, W.: Maximum principle for the finite element solution of time-dependent anisotropic diffusion problems. Numer. Methods Partial Differ. Equ. 29(6), 1963-1985 (2013)

21. Mincsovics, M.E.: Discrete and continuous maximum principles for parabolic and elliptic operators. J. Comput. Appl. Math. 235, 470-477 (2010)

22. Nakshatrala, K.B., Nagarajan, H., Shabouei, M.: A numerical methodology for enforcing maximum principles and the non-negative constraint for transient diffusion equations. Commun. Comput. Phys. 19(1), 53-93 (2016)

23. Pao, C.V., Ruan, W.H.: Positive solutions of quasilinear parabolic systems with nonlinear boundary conditions. J. Math. Anal. Appl. 333, 472-499 (2007)

24. Varga, R.: On a discrete maximum principle. SIAM J. Numer. Anal. 3(2), 355-359 (1966)

25. Vejchodský, T.: On the nonnegativity conservation in semidiscrete parabolic problems. In: Křížek, M., Neittaanmäki, P., Korotov, S., Glowinski, R. (eds.) Conjugate Gradient Algorithms and Finite Element Methods. Scientific Computation. Springer, Berlin (2004)

26. Vejchodský, T., Korotov, S., Hannukainen, A.: Discrete maximum principle for parabolic problems solved by prismatic finite elements. Math. Comput. Simul. 80(8), 1758-1770 (2010)

27. Vejchodský, T., Šolín, P.: Discrete maximum principle for higher-order finite elements in 1D. Math. Comput. 76(260), 1833-1846 (2007)

28. Wang, J., Zhang, R.: Maximum principles for $P 1$-conforming finite element approximations of quasilinear second order elliptic equations. SIAM J. Numer. Anal. 50(2), 626-642 (2012)

Publisher's Note Springer Nature remains neutral with regard to jurisdictional claims in published maps and institutional affiliations. 